International Journal of Applied Mathematical Research, 1 (4) (2012) 604-610 (C)Science Publishing Corporation

www.sciencepubco.com/index.php/IJAMR

\title{
Spatiotemporal Dynamics of Two Dimensional Logistic Maps Over a Complex Network
}

\author{
Santinath Pal ${ }^{1}$ and Anindita Tarai ${ }^{2}$ \\ ${ }^{1}$ Department of Mathematics, Tantigeria High School, \\ Paschim Medinipur, West Bengal, India. \\ Email: mathsanti@gmail.com \\ 2 Department of Mathematics Aligunj R. R. B. High School, \\ Paschim Medinipur, West Bengal, India. \\ Email: swarup_p@yahoo.com
}

\begin{abstract}
We investigate the spatiotemporal dynamics of two dimensional logistic maps over a complex network. The complex network is an one dimensional dynamic random network. The local on site map is chosen as the fully chaotic two species logistic map. Species of different nodes interact with symbiosis, predator-prey and competition. We study synchronization behaviour of the ring network under the above three types of interactions. We investigated the effect of coupling strength keeping rewiring probability $p$ fixed. Interesting observations are presented with figures.
\end{abstract}

Keywords: Coupled map lattice, networks, spatiotemporal dynamics, logistic maps, synchronization.

\section{Introduction}

In recent years, complex networks have provided an increasingly challenging framework for the study of collective behaviors in complex systems, based on the interplay between the wiring architecture and the dynamical properties of the coupled units [1]. In recent years it has become evident that modelling 
large interactive systems by finite dimensional lattices on one hand, and fully random networks on the other, is inadequate, as various networks, ranging from collaborations of scientists to metabolic networks, do not fit in either paradigm [2]. In fact many systems of biological, technological and physical significance are better described by randomizing some fraction of the regular links [3]. In this paper, we will study the spatiotemporal dynamics of coupled map latices(CMLs), with some of its coupling connections rewired randomly[5].

R. Lopez Ruiz et.al. [4] had assumed that two species $\left(x_{n}, y_{n}\right)$ are living on an island and the island has not any contact with the exterior world. They also assumed that for living species there is no possibility to migrate to a new land with affordable resources. Each species evolves following a logistic type dynamics,

$$
\begin{aligned}
x_{n+1} & =\mu_{x}\left(y_{n}\right) x_{n}\left(1-x_{n}\right) \\
y_{n+1} & =\mu_{y}\left(x_{n}\right) y_{n}\left(1-y_{n}\right)
\end{aligned}
$$

The interaction between species causes the growth rate $\mu(z)$ to vary with time, and $\mu(z)$ depends on the population size of the others and on a positive constant $\lambda$ which measures the strength of the mutual interaction. They choose the growth rate as a linear increasing $\mu_{1}$ or decreasing $\mu_{2}$ function expanding at the parameter interval where the logistic map shows nonvanishing population, that is $\mu \in(1,4)$. They assumed

$$
\begin{array}{r}
\mu_{1}(z)=\lambda(3 z+1) \\
\mu_{2}(z)=\lambda(-3 z+4)
\end{array}
$$

They proposed the following three types of coupling. Firstly, the symbiosis between species, it was modelled by the symmetrical coupling meaning a mutual interacting benifit, with $\mu_{x}=\mu_{y}=\mu_{1}$. Secondly, the predator-prey interaction, it is based on the benifit / damage relationship established between the predator and prey, respectively, then $\mu_{x}=\mu_{1}$ and $\mu_{y}=\mu_{2}$. Lastly, the competition between species causes the contrary symmetrical coupling, then $\mu_{x}=\mu_{y}=\mu_{2}$.

In this work, we assume that population of different islands can diffuse i.e. some fraction of population can migrate from one island to other islands with time. We have investigated the synchronization behaviour of the coupled two dimensional logistic maps over a ring network under different coupling strengths. We have also investigated the effects of different type of random connections in the network on synchronization property of the network. We consider logistic maps with symbiosis, predator-prey and competition interactions. 


\section{$2 \quad$ Model}

Specifically, we consider an one dimensional ring of coupled two dimensional logistic maps. The sites are denoted by integers $i=1,2, \ldots, N$, where $N$ is the linear size of the lattice. On each site a continuous state variable denoted by $x_{n}(i), y_{n}(i)$, is defined which corresponds to the physical variable of interest. The evolution of this lattice, under standard nearest neighbour interactions, in discrete time $\mathrm{n}$ is given by

$$
\begin{aligned}
& x_{n+1}(i)=(1-\epsilon) f\left(x_{n}(i), y_{n}(i)\right)+\frac{\epsilon}{2}\left\{x_{n}(i+1)+x_{n}(i-1)\right\} \\
& y_{n+1}(i)=g\left(x_{n}(i), y_{n}(i)\right)
\end{aligned}
$$

The strength of coupling is given by $\epsilon$. Now we will consider the above system with its coupling connections rewired randomly in varying degrees, and try to determine what dynamical properties are significantly affected by the way connections are made between elements. In our study, at every update we will connect a fraction $\mathrm{p}$ of randomly chosen sites in the lattice, to two other random sites, instead of their nearest neighbours. That is, we will replace a fraction $p$ of nearest neighbour links by random connections. That is, with probability $p$ the dynamical equations of the system are

$$
\begin{aligned}
& x_{n+1}(i)=(1-\epsilon) f\left(x_{n}(i), y_{n}(i)\right)+\frac{\epsilon}{2}\left\{x_{n}(\xi)+x_{n}(\eta)\right\} \\
& y_{n+1}(i)=g\left(x_{n}(i), y_{n}(i)\right)
\end{aligned}
$$

where $\xi$ and $\eta$ are random integers drawn from a uniform distribution in the set of integers $\{1,2,3, \ldots, N\}$, and with $(1-p)$ probability the dynamical equatn of the systems are

$$
\begin{aligned}
& x_{n+1}(i)=(1-\epsilon) f\left(x_{n}(i), y_{n}(i)\right)+\frac{\epsilon}{2}\left\{x_{n}(i+1)+x_{n}(i-1)\right\} \\
& y_{n+1}(i)=g\left(x_{n}(i), y_{n}(i)\right)
\end{aligned}
$$

The case of $p=0$ corresponds to the usual nearest neighbour interaction, while $p=1$ corresponds to completely random coupling . This scenario is much like small world networks at low $p$.

\subsection{Symbiosis}

When two species $\left(x_{n}, y_{n}\right)$ interect symbiotically the spatiotemporal evolution of the lattice, under random interactions, in discrete time $\mathrm{n}$ is given by the following. With probability $p$ the evolution equations are

$$
\begin{aligned}
& x_{n+1}(i)=(1-\epsilon) \lambda\left(3 y_{n}+1\right) x_{n}\left(1-x_{n}\right)+\frac{\epsilon}{2}\left\{x_{n}(\xi)+x_{n}(\eta)\right\} \\
& y_{n+1}(i)=\lambda\left(3 x_{n}+1\right) y_{n}\left(1-y_{n}\right)
\end{aligned}
$$


and with $(1-p)$ probability the dynamical equation of the systems are

$$
\begin{aligned}
& x_{n+1}(i)=(1-\epsilon) \lambda\left(3 y_{n}+1\right) x_{n}\left(1-x_{n}\right)+\frac{\epsilon}{2}\left\{x_{n}(i+1)+x_{n}(i-1)\right\} \\
& y_{n+1}(i)=\lambda\left(3 x_{n}+1\right) y_{n}\left(1-y_{n}\right)
\end{aligned}
$$

\subsection{Predator-Prey}

Let us think now that one of the species $x_{n}$ be a predator and $y_{n}$ be a prey. In this case, with probability $p$ the evolution equations are

$$
\begin{aligned}
& x_{n+1}(i)=(1-\epsilon) \lambda\left(3 y_{n}+1\right) x_{n}\left(1-x_{n}\right)+\frac{\epsilon}{2}\left\{x_{n}(\xi)+x_{n}(\eta)\right\} \\
& y_{n+1}(i)=\lambda\left(-3 y_{n}+4\right) y_{n}\left(1-x_{n}\right)
\end{aligned}
$$

and with $(1-p)$ probability equations of the systems follow the following are

$$
\begin{aligned}
& x_{n+1}(i)=(1-\epsilon) \lambda\left(3 y_{n}+1\right) x_{n}\left(1-x_{n}\right)+\frac{\epsilon}{2}\left\{x_{n}(i+1)+x_{n}(i-1)\right\} \\
& y_{n+1}(i)=\lambda\left(-3 y_{n}+4\right) y_{n}\left(1-x_{n}\right)
\end{aligned}
$$

\subsection{Competition}

Let us suppose now two species $\left(x_{n}, y_{n}\right)$ evolving under a competative interaction. Then spatiotemporal evolution of the lattice are governed by the following equations

$$
\begin{aligned}
& x_{n+1}(i)=(1-\epsilon) \lambda\left(-3 y_{n}+4\right) x_{n}\left(1-x_{n}\right)+\frac{\epsilon}{2}\left\{x_{n}(\xi)+x_{n}(\eta)\right\} \\
& y_{n+1}(i)=\lambda\left(-3 y_{n}+4\right) y_{n}\left(1-x_{n}\right)
\end{aligned}
$$

and with $(1-p)$ probability it are the following

$$
\begin{aligned}
& x_{n+1}(i)=(1-\epsilon) \lambda\left(-3 y_{n}+4\right) x_{n}\left(1-x_{n}\right)+\frac{\epsilon}{2}\left\{x_{n}(i+1)+x_{n}(i-1)\right\} \\
& y_{n+1}(i)=\lambda\left(-3 y_{n}+4\right) y_{n}\left(1-x_{n}\right)
\end{aligned}
$$

Where the positive parameter $\lambda$ expresses the strength of the mutual competative interaction.

\section{$3 \quad$ Results and Discussions}

We draw bifurcation diagram for single simbiotic logistic map in figure-1(a). Bifurcation diagram for the lattice of $\operatorname{simbiotic} \operatorname{logistic} \operatorname{maps}(\lambda=1.02)$ with nearest neighbour coupling is shown in figure-1(b), with random coupling with 
(a)

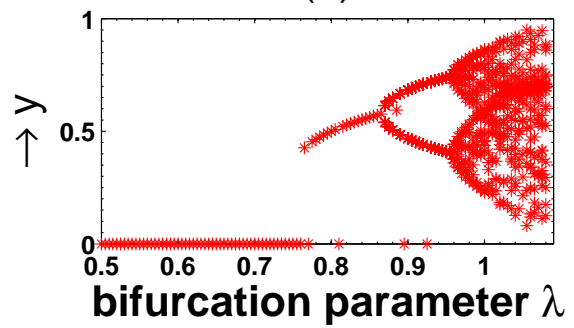

(c)

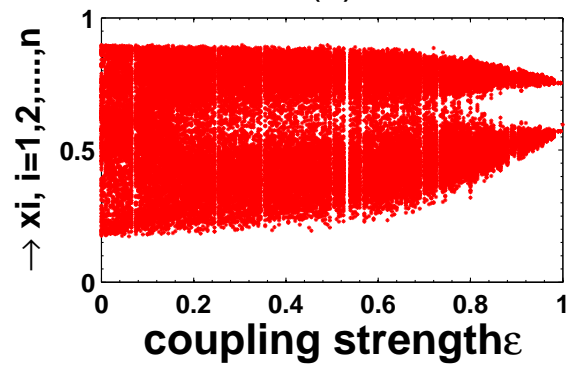

(b)

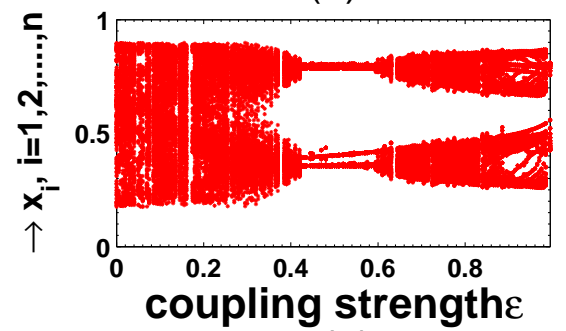

(d)

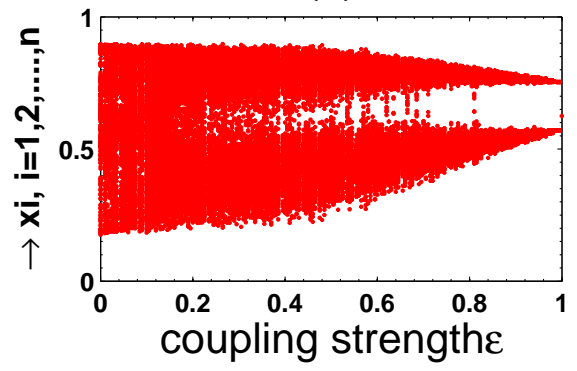

Figure 1: Bifurcation diagram of (a) single simbiotic logistic map, (b) the lattice with probability $p=0.0,(\mathrm{c})$ of the lattice with probability $p=0.5,(\mathrm{~d})$ of the lattice with probability $p=1.0$.

(d)

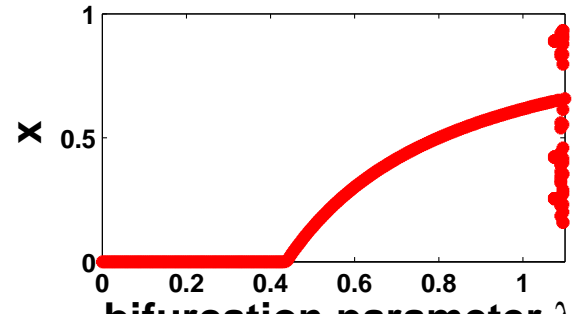

(c)

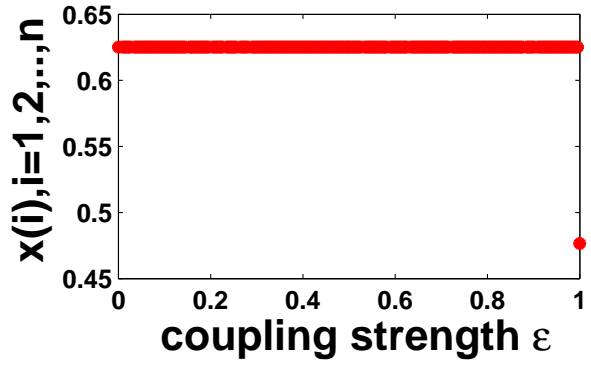

(D)

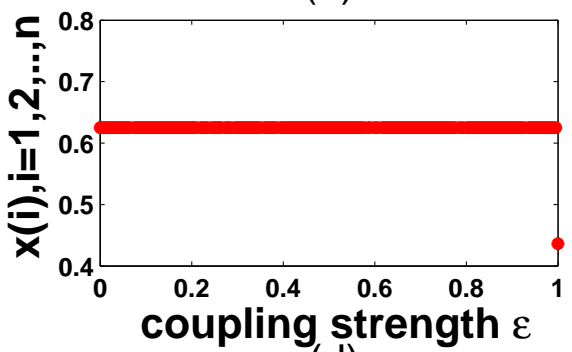

(d)

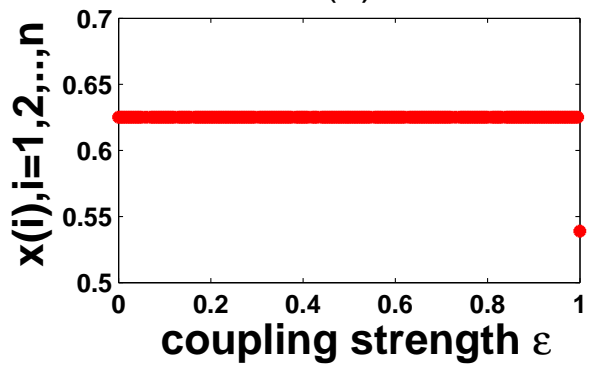

Figure 2: Bifurcation diagram of (a) single predator prey logistic map,(b) the lattice with probability $p=0.0,(\mathrm{c})$ of the lattice with probability $p=0.5,(\mathrm{~d})$ of the lattice with probability $p=1.0$. 

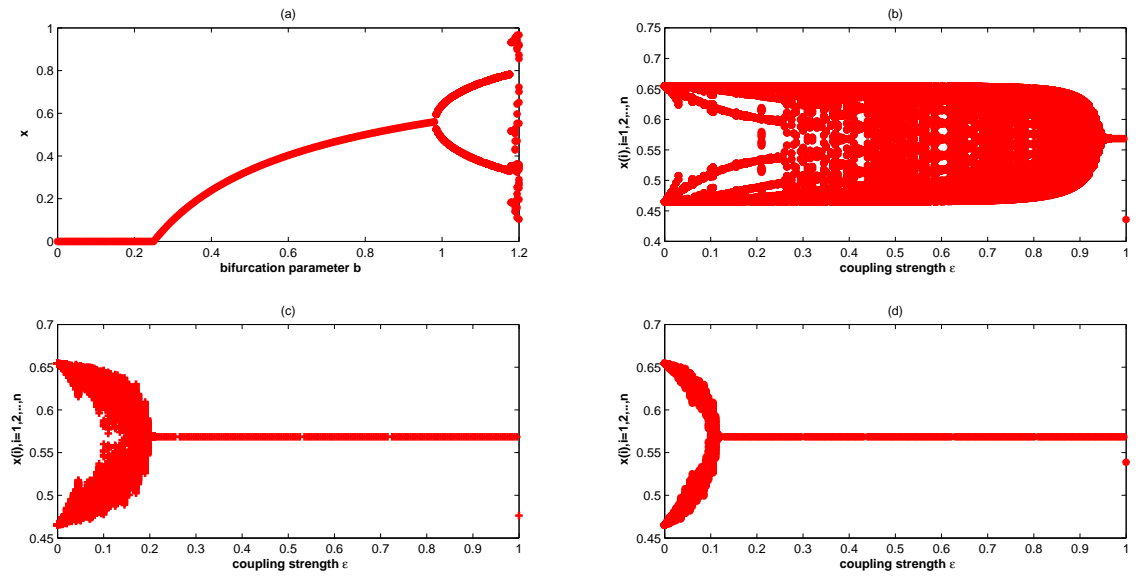

Figure 3: Bifurcation diagram of (a) single competetive logistic map,(b) the lattice with probability $p=0.0,(\mathrm{c})$ of the lattice with probability $p=0.5,(\mathrm{~d})$ of the lattice with probability $p=1.0$.

randomness $p=0.5$ is shown in figure-1(c) and for completely random interaction is shown in figure-1(d). We observe synchronization for nearest neighbour coupling only. We draw bifurcation diagram for single predator-prey type logistic map in figure-2(a). Bifurcation diagram for the lattice of predatorprey type logistic maps $(\lambda=1.02)$ with nearest neighbour coupling is shown in figure-2(b), with random coupling with randomness $p=0.5$ is shown in figure-2(c) and for completely random interaction is shown in figure-2(d). We observe stable co-existence of predator-prey species in this case. We draw bifurcation diagram for single competetive logistic map in figure-3(a). Bifurcation diagram for the lattice of competetive logistic maps $(\lambda=1.01)$ with nearest neighbour coupling is shown in figure-3(b), with random coupling with randomness $p=0.5$ is shown in figure-3(c) and for completely random interaction is shown in figure-3(d). We observe synchronization property increases with randomness here. This type of network will be usefull to devise control methods for spatially extended prey-predator systems. Our study suggest that natural regularizing mechanisms in physical and biological systems occur due to complex interactions.

\section{ACKNOWLEDGEMENTS}

We are grateful to the Editor for constructive comments. 


\section{References}

[1] S. Boccaletti, V. Latora, Y. Moreno, M. Chavez and D.U. Hwang, Complex Networks: Structure and dynamics, Phys. Reports 424 (2006) 175308.

[2] S. Poria, M.D. Srimali, S. Sinha, Enhancement of spatiotemporal regularity in an optimal window of random coupling, Phys. Rev. E 78, 035201R (2008).

[3] D.J. Watts and S.H. Strogatz, Collective dynamics of small-world networks, Nature (London) 393 (1998) 440-442.

[4] R.Lopez-Ruiz and D.Fournier-Prunaret, Three Logistic models for the two species interaction: Symbiosis,Predator-prey and Competition, Nonlinear Science 0406020v1 (2004).

[5] S.Pal and S.Poria, Spatiotemporal Dynamics of Coupled Ikeda Maps Over a Ring Network, International Journal of Applied Mathematical Research, 1 (4) (2012) 383-390. 